Submitted to ApJ on Dec.11, 2015; Revised on Feb.8, 2016; Accepted on Feb.23, 2016

Preprint typeset using $\mathrm{IAT}_{\mathrm{E} X} \mathrm{X}$ style emulateapj v. 5/2/11

\title{
TRANSITING PLANET CANDIDATES BEYOND THE SNOW LINE DETECTED BY VISUAL INSPECTION OF 7557 KEPLER OBJECTS OF INTEREST
}

\author{
Sho Uehara $^{1}$, Hajime Kawahara ${ }^{2,3}$, Kento Masuda ${ }^{4}$, Shin'ya Yamada ${ }^{1}$, and Masataka Aizawa ${ }^{4}$ \\ Submitted to ApJ on Dec.11, 2015; Revised on Feb.8, 2016; Accepted on Feb.23, 2016
}

\section{ABSTRACT}

We visually inspected the light curves of 7557 Kepler Objects of Interest (KOIs) to search for single transit events (STEs) possibly due to long-period giant planets. We identified 28 STEs in 24 KOIs, among which 14 events are newly reported in this paper. We estimate the radius and orbital period of the objects causing STEs by fitting the STE light curves simultaneously with the transits of the other planets in the system or with the prior information on the host star density. As a result, we found that STEs in seven of those systems are consistent with Neptune- to Jupiter-sized objects of orbital periods ranging from a few to $\sim 20 \mathrm{yr}$. We also estimate that $\gtrsim 20 \%$ of the compact multi-transiting systems host cool giant planets with periods $\gtrsim 3 \mathrm{yr}$ on the basis of their occurrence in the KOIs with multiple candidates, assuming the small mutual inclination between inner and outer planetary orbits. Subject headings: planets and satellites: detection — planets and satellites: individual (KOI-671, KOI-1421, KOI-2525, KOI-847, KOI-1108, KOI-693, KOI-435) — techniques: photometric

\section{INTRODUCTION}

About twenty years have passed since the first discovery of exoplanets using the radial velocity method (Mavor \& Queloz 1995). Now the radial velocity surveys reach long-period exoplanets around and beyond the snow line, including Jupiter-analogues around sunlike stars (e.g. Boisse et al. 2012; Marmier et al. 2013; Bedell et al. 2015; Rowan et al. 2015). How do we further characterize those cool gas and ice giants? While direct imaging is a promising approach to characterize them in near future (e.g. Hagelberg 2010; Salter et al. 2014), transiting long-period giant planets (LPGs), on which the present paper focuses, are also important for probing the planetary system architecture beyond the snow line. Indeed, detailed information on the system architecture, including the statistical properties of resonance and the mutual orbital inclination, has already been obtained for the compact multi-transiting systems (orbital periods $\lesssim 1 \mathrm{yr}$ ) discovered by the Kepler spacecraft (e.g. Lissauer et al. 2011; Fabrycky et al. 2014; Winn \& Fabrycky 2015). Transiting LPGs will also provide the opportunity to characterize both the interior structure and atmospheric compositions of the cool giant planets with transmission spectroscopy, as already demonstrated for the solar-system planets. For example, the observed transmission spectra of Jupiter (Montañés-Rodríguez et al. 2015) and Saturn (Dalba et al. 2015) exhibit clear features of atmospheric molecules such as methane.

Despite their importance, it is extremely challenging to find the transiting LPGs at all. Since the transit prob-

Electronic address: sho_u@phys.se.tmu.ac.jp

${ }^{1}$ Department of Physics, Tokyo Metropolitan University, Tokyo 192-0397, Japan

2 Department of Earth and Planetary Science, The University of Tokyo, Tokyo 113-0033, Japan

${ }^{3}$ Research Center for the Early Universe, School of Science, The University of Tokyo, Tokyo 113-0033, Japan

${ }^{4}$ Department of Physics, The University of Tokyo, Tokyo 1130033, Japan ability of LPGs is quite low $(\sim 0.1 \%)$, it is hopeless to search for them in the sample of LPGs characterized with radial velocities (RVs). Even utilizing the space-mission data as obtained by Kepler, transiting LPGs can hardly be detected with the usual periodicity analysis because their orbital periods are typically beyond the mission lifetime. Nevertheless, they can still be detected through the single transit events (STEs), which occur only once in the 4-year observational span and thus may have been missed by the pipeline 5

In this paper, we perform a uniform search for the STEs in the Kepler data by visual inspection and report on the discovery of seven candidates of transiting LPGs. We focus on the targets with already known transit signals (i.e. Kepler Objects of Interest, KOIs) mainly for the two reasons. First, it is more likely to find transiting LPGs for those systems because the orbital planes of LPGs are presumably aligned with those of the inner planets, at least, to some extent. Second, we can estimate the orbital periods of those transiting LPGs even from the single transit, if found, by fitting their light curves simultaneously with those of the inner planets. We will demonstrate that such an estimate is indeed possible and also useful for discussing the system architecture.

The rest of the paper is organized as follows. $\sqrt{2}$ presents the methods for finding the STEs and the analysis for estimating their geometric parameters, especially the orbital period. 3 describes the features of the individual STEs in more detail, and classifies them based on the likelihood to be genuine planets. Implications of our finding for the statistical property of LPGs are also briefly discussed in 4 .

\section{IDENTIFICATION OF SINGLE TRANSIT EVENTS AND ORBITAL PERIOD ESTIMATION}

${ }^{5}$ Note that the independent work by Wang et al. (2015), published during the preparation of this manuscript, is based on a similar motivation to ours. The STEs in five systems we identified (KOI-4307, KOI-3349, KOI-847, KOI-1168, and KOI-3145) have also been reported in their paper. 
Let us first summarize the STEs we identified before the detailed description of the analysis. Table 1 reports 28 STEs we identified in 24 KOIs. We analyzed 16 STE light curves in 14 of those systems that are not the clear false positives and estimated the parameters including the orbital period and radius ratio (Table 2). As a result, we found seven systems exhibiting STEs consistent with the planetary transit ( $\S 3$ in detail); their orbital architectures are illustrated in Figure 1. In this section, we describe the details of the STE identification (\$2.1) and light-curve analyses $(\S 2.2)$.

\subsection{Identification of Single Transiting Events (STEs)}

We analyzed the long cadence fluxes of the Pre-search Data Conditioning component of the Kepler pipeline (PDCSAP) of 7557 KOIs, which were available on the NASA Exoplanet Archive at the time of June 4th, 2015. We searched for STEs by visual inspection of all those PDCSAP fluxes and identified 28 STEs in 24 KOIs as shown in Table 1. Here the fading events that are not observed in the SAP data but only seen in the PDCSAP data are excluded because we find that the correction by the PDC pipeline sometimes leads to artificial dips in the light curves 6 Although it is admittedly difficult to quantify the detection limit of our visual inspection, we believe that transits deeper than $\sim 0.1 \%$ and lasting longer than 5-50 hours have been detected.

Among the 28 STEs in Table 1, 14 have never been reported in the literature; they are marked with "new" in the parentheses. KOI numbers designated by the Kepler team are listed for the other seven events. When more than one STEs are found in one system, they are reported separately (two in KOI-847, KOI-1168, and KOI-6378; three in KOI-1032).

\subsection{Geometric Parameters of STE Candidates}

To further characterize the planet candidates causing STEs (hereafter "STE candidates"), we fit the STE light curves assuming that STE candidates are not due to contamination but orbiting the KOIs for which we found STEs on circular orbits. As discussed below, this assumption allows us to estimate orbital periods of the STE candidates even from only one transit. Here we exclude the systems designated as false positives in the KOI catalog and KOI-1032 exhibiting signatures of the CCD cross talk 7 because the above assumption is less sound for them. We also examined the target pixel files of the remaining targets visually and excluded the STE of KOI-3145, whose depths are different in neighboring pixels and thus likely to be due to contamination from a nearby star (see also Wang et al. 2015). These criteria leave us with 16 STEs in 14 systems, which are listed in Table 2 along with their estimated parameters.

\subsubsection{Principle}

While we use the Markov-Chain Monte Carlo (MCMC) method to determine the system parameters, here we an-

${ }^{6}$ For instance, PDCSAP data of KOI-6469 (KIC 4912589) exhibits a dip at BJD $-2454833=613.5$, which does not exist in the SAP data.

7 This system is classified as a possible false positive on Kepler Community Follow-up Observing Program (CFOP) webpage as well. alytically show how the orbital period of the STE candidate is derived with the information on the mean stellar density either from the light curves of inner transiting planets with known orbital periods or from the follow-up observations of the host star.

Assuming a circular orbit, the total and full transit durations (denoted by $t_{T}$ and $t_{F}$, respecitvely) are given by (e.g. Winn 2010)

$$
\begin{aligned}
t_{T} & =\frac{P}{\pi} \sin ^{-1}\left(\frac{R_{*}}{a} \sqrt{\frac{(1+k)^{2}-b^{2}}{\sin i}}\right), \\
t_{F} & =\frac{P}{\pi} \sin ^{-1}\left(\frac{R_{*}}{a} \sqrt{\frac{(1-k)^{2}-b^{2}}{\sin i}}\right),
\end{aligned}
$$

where $k \equiv R_{p} / R_{*}$ indicates the ratio of the planetary radius to the stellar radius, $i$ is the orbital inclination, and $b$ is the impact parameter. Neglecting the terms of $\mathcal{O}\left(\left(R_{*} / a\right)^{3}\right)$ and higher, we obtain

$$
\frac{R_{*}}{a}=\frac{\pi}{2 \sqrt{k}} \frac{\sqrt{t_{T}^{2}-t_{F}^{2}}}{P} .
$$

Combined wtih Kepler's third law, Equation (3) yields the orbital period of an STE candidate in terms of its transit shape and the mean stellar density $\rho_{*}$ (Seager \& Mallén-Ornelas 2003):

$$
P=\frac{\pi G}{32} \frac{M_{*}}{R_{*}^{3}}\left(\frac{t_{T}^{2}-t_{F}^{2}}{k}\right)^{\frac{3}{2}} \simeq \frac{\pi^{2} G}{3} \rho_{*}\left(\frac{T \tau}{k}\right)^{3 / 2}
$$

where $M_{*}$ and $R_{*}$ are the stellar mass and radius. We also defined $T \equiv \frac{1}{2}\left(t_{T}+t_{F}\right)$ and $\tau \equiv \frac{1}{2}\left(t_{T}-t_{F}\right)$ and assumed $\tau \ll T$.

If the inner transiting planet(s) is known in the system, Equation (4) can be used to constrain $\rho_{*}$ from its transit shape because $P$ is already determined for the planet(s). In this case, the orbital period of the STE candidate $P_{s}$ is given by

$$
P_{s}=\left(\frac{k_{i} T_{s} \tau_{s}}{k_{s} T_{i} \tau_{i}}\right)^{\frac{3}{2}} P_{i}
$$

where the subscripts $s$ and $i$ denote the quantities for the STE candidate and inner planet, respectively. This is the case for the 10 systems except for KOI-1421, 1208, 1174, and 1096 in Table 2. We checked that the analytic estimate in Equation (5) is indeed consistent with the MCMC results for these systems. Even if inner transiting planets are not known, the prior knowledge on $\rho_{*}$ from the color photometry can also be used to constrain $P_{s}$, although it may be less reliable than the dynamical value as obtained in the previous case; this method is adopted for KOI-1421, 1208, 1174, and 1096.

\subsubsection{MCMC Fit to the Observed STE Light Curves}

For the 10 systems except for KOI-1421, 1208, 1174, and 1096, we fit the STE light curves simultaneously with the phase-folded transit light curves of the other planet candidates in the system. We basically analyzed the long-cadence PDCSAP fluxes except for the inner transits of KOI-671 and KOI-435, for which shortcadence fluxes were used. We used PyTransit package (Parviainen 2015) to generate the transit light curve 
based on Mandel \& Agol (2002) model for the quadratic limb-darkening law. The effect of binning was taken into account by supersampling for the long-cadence data sampled at 30 minutes. Constraints on the parameters were obtained by the MCMC sampling using emcee package (Foreman-Mackey et al. 2013) following the standard $\chi^{2}$ minimization using the Levenberg-Marquardt method (mpfit by Markwardt 2009). The likelihood for the MCMC sampling $\mathcal{L}$ was computed as $\mathcal{L} \propto \exp \left(-\chi^{2} / 2\right)$, where $\chi^{2}$ is a sum of the standard chi-squared for each planet's transit, and we adopt non-informative priors unless otherwise noted.

Transits of the inner planets were processed as follows. We first detrended the light curves from each quarter using the second-order spline interpolation, after masking the known transits based on the ephemeris and duration in the KOI catalog. Baseline fluxes during the transit were determined by the linear interpolation between the two ends of the masked region. The detrended transits were folded at the orbital period given in the KOI catalog and averaged into one-minute bins. The value and error of the binned flux are given by the mean and its standard deviation of the values in each bin. The smoothing parameter of the spline was chosen so that the depth of the phase-folded transit be consistent with the catalog value. The STE light curves were deterened in a similar manner except that the smoothing parameter was chosen to be 0.1 days and that the endpoints of the linear interpolation were adjusted so that the resulting detrend light curve be not too asymmetric.

The fitting parameters in this case are the sum of two coefficients for the quadratic limb-darkening law $u_{1}+u_{2}$ and $\rho_{*}$ as the parameters common to all the planets in the same system; time of transit center $T_{c}, \cos i, k$, and normalization of the flux $c$ for each of the inner transiting planets; and $T_{c}, \cos i, k, c$, and orbital period $P_{s}$ for STE candidates. The difference of the limb-darkening coefficients $u_{1}-u_{2}$ were fixed to be the linearly interpolated values based on Sing (2010), and the orbital periods of the inner planets were fixed at the values given in the KOI catalog. In MCMC fitting, we adopt the prior distributions uniform in $u_{1}+u_{2}, \log \rho_{*}, T_{c}, \cos i, \log k, c$, and $\log P_{s}$. We assumed circular orbits for both inner candidates and STE candidates.

For KOI-1421, 1208, 1174, and 1096, we fit the STE light curve alone, imposing the Gaussian prior on $\rho_{*}$ based on the value at CFOP. The fitting parameters in this case are the two stellar parameters $u_{1}+u_{2}$ and $\rho_{*}$, and $T_{c}, \cos i, k, c$, and $P_{s}$ of the STE candidate.

In the analyses above, we neglect the effect of possible stellar multiplicity. If the target star has an unidentified companion star, for instance, the planetary radii can be underestimated due to the dilution. We note, however, that this possibility is essentially ruled out for most of our main candidates discussed in Section 3.1.

The best-fit models and constraints on the parameters of STE candidates are summarized in Figure 2 and Table 2. All the transits of the inner candidates simultaneously fitted with STEs are listed in Appendix. On the basis of the inferred orbital period and radius of STE candidates, we found that STEs in seven systems are consistent with the planetary transit, as will be detailed in $\$ 3$. Architectures of these seven systems are illustrated in Figure 1 We also plotted each STE candidate on the $R_{p}-P$ plane with all known KOI candidates in Figure 3, The figure shows that STE candidates we found are all likely to be gas/ice giants beyond the snow line.

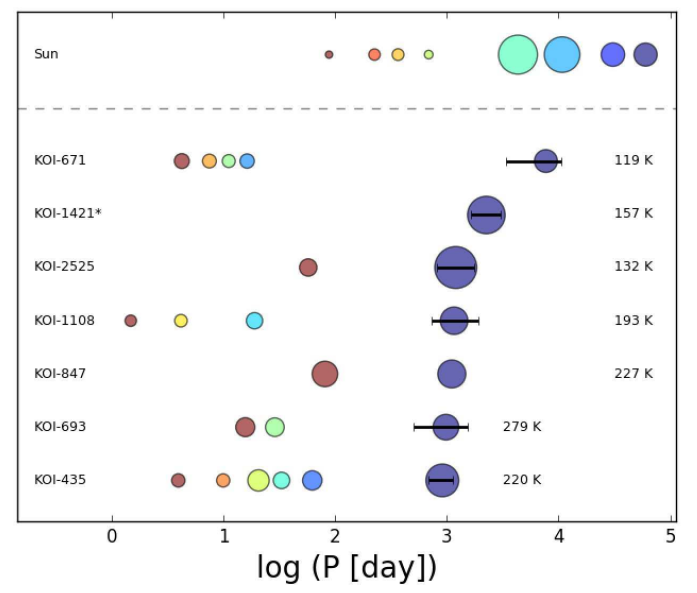

Figure 1. Architectures of the KOI systems for which we identified STEs consistent with transiting LPGs (listed in the upper part of Table 2). The size of the circles are proportional to the estimated planet radius. The STE candidates are the rightmost circles in each row, illustrated with error bars for the estimated period and its radiative equilibrium temperature. Note that the orbital period determined from the interval of the two STEs is adopted for KOI-847. Our solar system is shown at the top for reference. The orbital period of the STE candidate in KOI-1421 (marked with an asterisk) is based on the stellar density provided by CFOP and may be less reliable than the others.

\section{CLASSIFICATION OF STE CANDIDATES AND DESCRIPTION OF INDIVIDUAL SYSTEMS}

On the basis of the fitting results, we classify the STE candidates in Table 2 into three categories:

1. candidates consistent with transiting LPGs,

2. candidates exhibiting anomalous orbital periods, which we call "misfit" singles, and

3. candidates likely to be eclipsing binaries (EBs).

In the following, we describe the details of the classification and comment on the properties of individual systems.

\subsection{STE Candidates Consistent with the Transiting $L P G s$}

Seven systems illustrated in Figure 1exhibit STEs consistent with the planetary transit. Namely, (1) their transit light curves are consistently explained with those of the inner transiting planets or stellar density $\rho_{*}$ in the KIC catalog, (2) their inferred radii are less than about the Jupiter radius, and (3) their inferred orbital periods are consistent with the absence of other transits in the exiting Kepler data (i.e. inferred $P_{s}$ is longer than

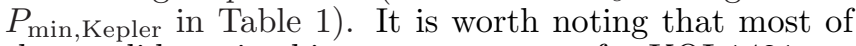
the candidates in this category except for KOI-1421 are in multi-planetary systems and thus likely to be genuine planets in the same systems (Lissauer et al. 2014). 


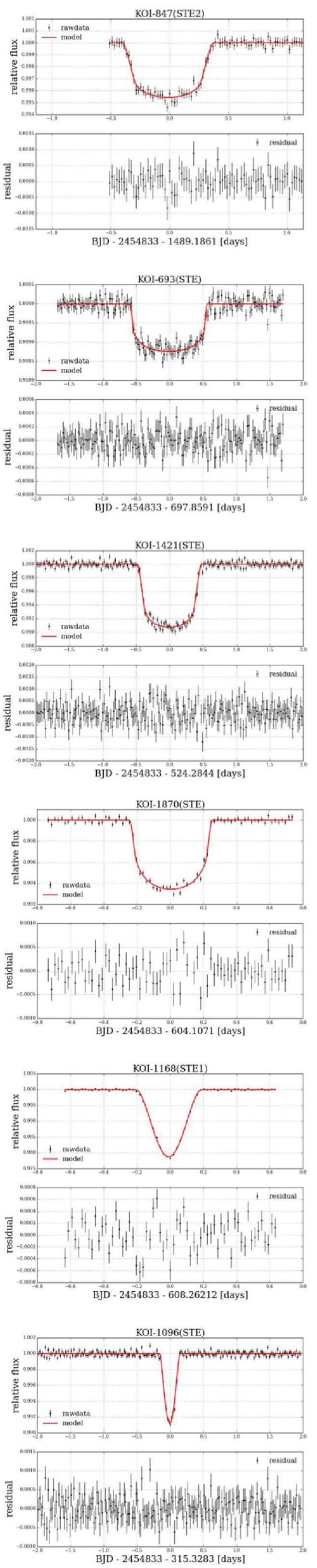
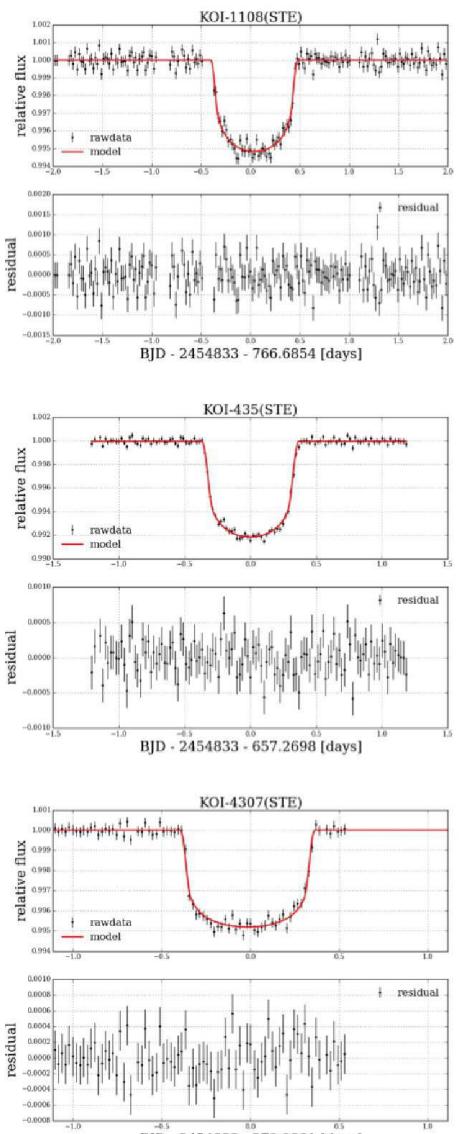

BjD - $2454833-279.9881$ days
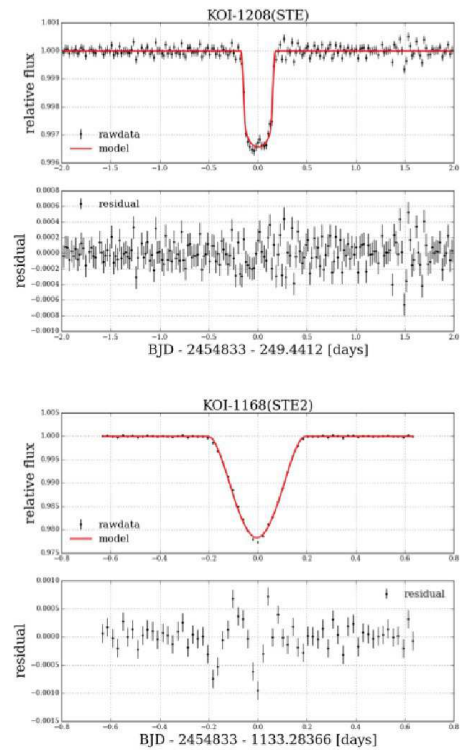

Figure 2. Light curves of the 16 STEs we analyzed. The solid red lines show the best-fit model obtained by $\chi^{2}$ minimization. 


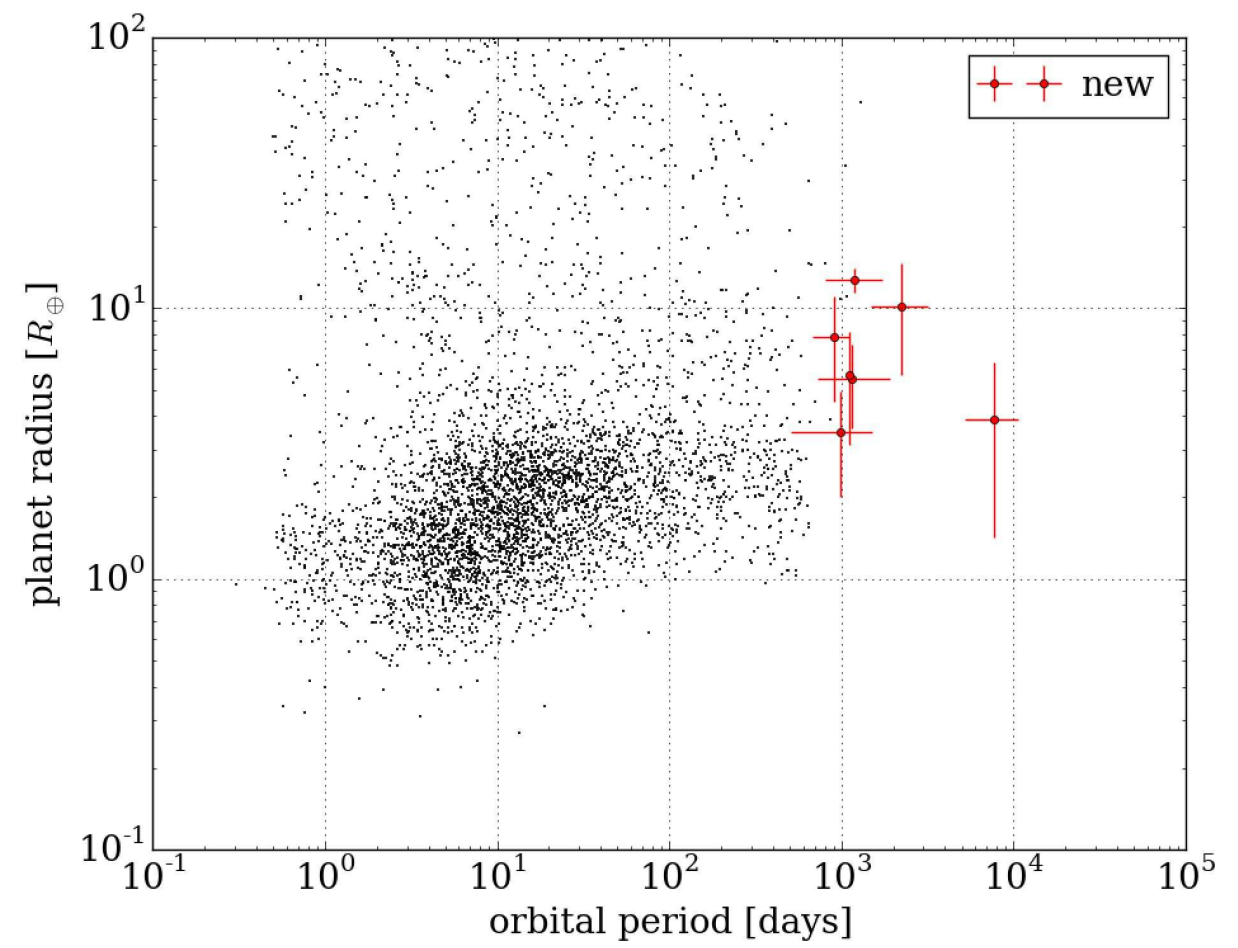

Figure 3. Properties of the seven STE candidates in Figure 1 on the period-radius plane (red dots with error bars). All known KOI candidates are also shown by small black dots. 


\section{KOI-847 (KIC 6191521)}

A Neptune-sized planet candidate with the period of 80.9 days (KOI-847.01) is already known in this system. We found additional two transit at $T_{c}($ BJD -2454833$)=$ 382.9428 and 1489.1858. 8 If these two transits originate from the same object, its orbital period $P_{s}$ is $1106.243 \pm$ 0.007 days. We individually fit each of the two events with the inner transit light curves and found the periods consistent with the above interval, $P_{s}=930_{-380}^{+430}$ days and $840_{-300}^{+380}$ days, respectively. In addition, the other transit parameters were also consistent with each other. The two STEs are therefore likely to be attributed to the same Neptune-sized planet with $P_{s}=1106.243 \pm$ 0.007 days.

\section{KOI-671 (KEPLER-208, KIC 7040629)}

KOI-671 is known as a compact multi-transiting system hosting four transiting planet candidates inside the orbit of Mercury (KOI-671.01-04). We found an STE at $T_{c}$ (BJD -2454833$)=786.7641$ consistent with a Neptune-sized planet of $R_{p}=3.9 \pm 2.5 R_{\oplus}$ and $P_{s}=$ $7700_{-2500}^{+2900}$ days. This $P_{s}$ is the longest among our STE candidates we found and is between those of Jupiter and Saturn.

\section{KOI-2525 (KIC 5942949)}

This system has one super-Earth candidate (KOI2525.01). We found an STE at $T_{c}(\mathrm{BJD}-2454833)=$ 1326.1614 days, for which we found $R_{p}=12.7 \pm 1.3 R_{\oplus}$ and $P=1200_{-390}^{+540}$ days from the MCMC analysis.

\section{KOI-1108 (KIC 3218908)}

KOI-1108 has a compact multi-transiting system with three super-Earth candidates within $P=18$ days (KOI1108.01-03). We found an STE by a Neptune-sized object $\left(R_{p}=5.5 \pm 1.9 R_{\oplus}\right)$ with $P=1160_{-430}^{+760}$ days.

\section{KOI-693 (KEPLER-214, KIC 8738735)}

This system harbors two confirmed super Earths Kepler-214b and c. We found one STE corresponding to a Neptune-sized object with $R_{p}=3.5 \pm 1.5 R_{\oplus}$ and $P=980_{-470}^{+520}$ days.

\section{KOI-435 (KEPLER-154, KIC 11709124)}

The system hosts two confirmed super-Earths/subNeptunes (Kepler-154a and b) and three planet candidates of similar radii. We found an STE due to a Saturnsized object at $T_{c}($ BJD -2454833$)=657.2698$, which is listed as KOI-435.02 in the KOI catalog. Our estimated radius and period are consistent with those in the catalog. The orbital period is best constrained for this STE candidate (except for KOI-847 exhibiting two STEs) due to the presence of multiple inner transiting planets and high signal-to-noise ratio.

\section{KOI-1421 (KIC 11342550)}

The STE we found is also listed in the KOI cata$\log$ as KOI-1421.01. Since this is the only transit signal known for the system, we fit the single STE light

8 These events are reported independently by Wang et al. (2015) as well. curve with a Gaussian prior on the mean stellar density $\rho_{*}=1.403 \pm 0.3668 \mathrm{~g} / \mathrm{cc}$, which is based on the CFOP value. As a result, we obtained $P=2230_{-740}^{+960}$ days and $R_{p}=10.2 \pm 4.5 R_{\oplus}$. The orbital period is consistent with the value given in the KOI catalog. As mentioned at the beginning of this subsection, this candidate has a higher false positive probability than the others because this system has no inner companion. However, we ruled out the companion identified at $11^{\prime \prime}$ away as a source of the fading event from inspection of the target pixel file.

\section{2. "Misfit" Singles: KOI-4307, KOI-3349, KOI-1870, KOI-1208, KOI-1174, and KOI-1096 - Eccentric Planets of False Positives?}

For the STEs in these systems, the orbital periods inferred from our fitting are shorter than $P_{\min , \text { Kepler in Ta- }}$ ble 1] which is the minimum orbital period required for the STE candidate to be consistent with the absence of other transit signals in the existing Kepler data. While the discrepancy may imply that they are false positives, it is also possible that the orbits of these STE candidates are eccentric, as shown below. For this reason, we still consider these "misfit" singles as planet candidates, though less promising than those listed in the previous subsection.

For simplicity, we consider the case where only the STE candidates have non-zero eccentricities, while the inner transiting objects are all on circular orbits. For eccentric orbits, the right-hand sides of $t_{T}$ and $t_{F}$ in Equations (1) and (2) are multiplied by $\sqrt{1-e^{2}} /(1+e \sin \omega)$, where $e$ is the eccentricity and $\omega$ is the argument of periastron (measured from the sky plane) of the STE candidate (e.g. equation (16) of Winn 2010). Thus, the orbital period of the STE candidate with non-zero eccentricity differs from the circular case by the factor of

$$
\alpha_{\mathrm{ecc}}=\left(\frac{1+e \sin \omega}{\sqrt{1-e^{2}}}\right)^{3} \leq\left(\frac{1+e}{1-e}\right)^{3 / 2}
$$

assuming the same $\rho_{*} \cdot 9$ Since $\alpha_{\text {ecc }}$ is larger than the ratio of $P_{\text {min, Kepler }}$ to $P_{s}$ obtained from the circular fit (Table 2), i.e., $\alpha_{\text {ecc }}>P_{\text {min,Kepler }} / P_{s}$, Equation (6) yields the minimum value of eccentricity required to explain the observation:

$$
e_{\min }=\frac{\left(P_{\min , \text { Kepler }} / P_{s}\right)^{2 / 3}-1}{\left(P_{\min , \text { Kepler }} / P_{s}\right)^{2 / 3}+1} .
$$


candidates are listed in Table 3, along with their values computed for the most conservative case (see the note in the table).

If (some of) these candidates are confirmed to be eccentric LPGs by follow-up observations, they can be interesting targets to understand the origin of hot Jupiters, as in the case of HD 80606 (Wu \& Murray 2003). In this regard, the most promising target is KOI-1208 with the Kepler magnitude of 13.6 (Table 1), for which a possible nearby companion has been detected and the eccentricity is estimated to be $\gtrsim 0.8$.

\footnotetext{
${ }^{9}$ While the joint fit including STE candidate's eccentricity may change $\rho_{*}$ as well, we neglect such rather small effects for the rough estimate here.
} 


\subsection{Candidate Likely to be an EB: KOI-1168}

The inferred radius of this STE candidate suggests that it is a stellar object rather than a planet. The good agreement between the parameters of the two STEs strongly implies that they are due to the same object. If this is indeed the case, this objects has $P_{s}=$ $525.0216 \pm 0.0006$ days. This system is also discussed in Wang et al. (2015), who also classified it as a likely false positive.

\section{DISCUSSION}

\subsection{Gap-like Structure}

In Figure 1 the systems with multiple inner planets (KOI-671, 1108, 693, and 435) exhibit a gap-like signature between the inner and STE candidates, as seen between the terrestrial and giant planets in the solar system (top row). While we cannot completely exclude the existence of such a gap in our sample, we suspect that it is due to the decrease in the transit probability with increasing orbital periods. Because of the low transit probability of a distant planet, transiting objects on wide orbits (say $P \gtrsim 10^{3}$ days) tend to be rare. This means that the system with one long-period transiting object detected, as in our sample, is unlikely to have another transiting object close to the detected one and exhibits a gap around the detected transiting object.

To demonstrate the above geometric effect, we performed a simple simulation of the transit detection of the multi-planetary system. We put 12 planets at a constant log-interval as in the first row of Figure 4. The semi-major axis over the host star radius, $a / R_{\star}$, of the outermost planet was chosen to be 420 , which corresponds to $P=10^{3}$ days or $a=1.96$ AU in the solar system. We simulated the transit observation of this system from many different directions uniformly distributed in $\cos i$, sampling the mutual inclinations of the inner planets with respect to the outermost one from the Rayleigh distribution with $\sigma=1.8$ (Fabrycky et al. 2014) in each run. From the resulting sample of transiting systems, we chose the systems where the transit of the outermost planet was detected and plotted their apparent architecture below the horizontal dashed line in Figure 4. One can actually see the gap-like structure between the outermost planets (red circles) and the inner planets (blue circles) in many cases.

\subsection{Occurrence Rate of the LPG in Compact Multi-transiting Systems}

As summarized in Table 4, the sample of 7557 KOIs we surveyed includes 695 systems with more than one inner transiting planet candidates, among which we detected four LPG candidates (see also Figure 1). These numbers can be used to estimate the occurrence rate of LPGs in compact multi-transiting systems on the premise that LPG orbits are likely to be well aligned with those of the inner multiple planets. The premise is based on the following argument. The planets in a compact multi-transiting system presumably have well-aligned orbits (Fabrycky et al. 2014), indicating that their orbital planes trace the original protoplanetary disk. Since an LPG in the same system formed in the same disk, its orbit is likely to be aligned with the inner ones as well.

The expected number of transiting LPGs, $n_{\mathrm{tLPG}}$, in

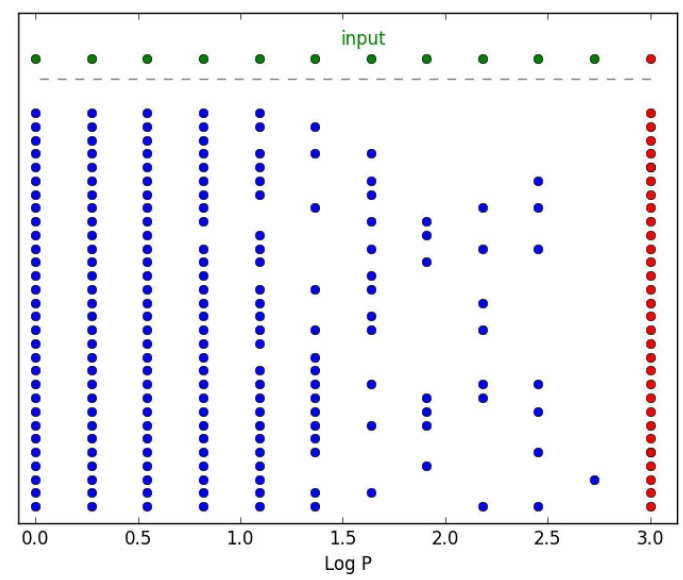

Figure 4. Simulated system architectures detected by transit observations. The top row shows the architecture of the input planetary system. The other rows plot the planets detected with simulated transit observations (blue circles) for the systems whose outermost planet (red circles) transits.

$N_{\text {cmulti }}$ compact multi-transiting systems is given by $n_{\mathrm{tLPG}} \simeq \frac{T_{\mathrm{obs}}}{P_{\mathrm{LPG}}} p($ tra $\mid \mathrm{LPG}$, cmulti $) \bar{n}\left(\mathrm{LPG} \mid\right.$ cmulti) $N_{\mathrm{cmulti}}$, where $\bar{n}(\mathrm{LPG} \mid$ cmulti) is the average number of LPGs per system and $p$ (tra|LPG, cmulti) is the transit probability of a given LPG, both under the existence of the inner compact multi-transiting system. The factor $T_{\mathrm{obs}} / P_{\mathrm{LPG}}$, where $T_{\mathrm{obs}}$ is the observing duration of $\mathrm{Ke}$ pler and $P_{\mathrm{LPG}}$ is the orbital period of a given LPG, takes into account the probability that the single transit of the LPG falls into the mission life time of Kepler. Since $p$ (tra|LPG, cmulti) depends on the mutual inclination of the LPG and the inner planets, the mutual inclination and occurrence rate $\bar{n}$ are usually degenerate (Tremaine \& Dong 2012).

As far as the mutual inclination between the LPG and inner-planet orbits is as small as $\sim R_{\star} / a_{\text {in }}$, we approximately have $p$ (tra $\mid \mathrm{LPG}$, cmulti $)=a_{\mathrm{in}} / a_{\mathrm{LPG}}$, where $a_{\mathrm{LPG}}$ and $a_{\text {in }}$ are the typical semi-major axes of the LPG and inner multi-transiting planets, respectively (see $\S 2.3$ of Ragozzine \& Holman 2010). Adopting $a_{\mathrm{LPG}}=2 \mathrm{AU}$ (corresponding to $P \simeq 10^{3}$ days) and $a_{\text {in }}=0.07 \mathrm{AU}$ (median of KOI candidates in multi-transiting systems), we obtain $p$ (tra|LPG, cmulti) $=0.035$, which yields $\bar{n}(\mathrm{LPG} \mid \mathrm{cmulti}) \simeq 0.2$ for $n_{\mathrm{t} \mathrm{LPG}}=4, N_{\text {cmulti }}=695$, $T_{\mathrm{obs}}=4 \mathrm{yr}$, and $P_{\mathrm{LPG}}=2200$ days (average of the seven systems in Figure 10 in Equation (8).

If the LPG has actually larger mutual inclination relative to the inner planets than assumed here $\left(\gtrsim R_{\star} / a_{\text {in }} \sim\right.$ $4^{\circ}$ ), the above estimate underpredicts the true occurrence rate 10 In addition, the above discussion assumes that the transiting LPG is $100 \%$ detected as long as it transits the host star during the Kepler observation. For these reasons, we conclude that $\bar{n}(\mathrm{LPG} \mid$ cmulti $) \simeq 0.2$ estimated above is a rough lower limit, and that about $20 \%$ or more of the compact multi-transiting systems

\footnotetext{
10 If the inclination of the outer LPG is completely random, for instance, $p($ tra $\mid \mathrm{LPG}$, cmulti $)=R_{\star} / a_{\mathrm{LPG}} \approx 0.002$ for $a_{\mathrm{LPG}}=2 \mathrm{AU}$ and $R_{\star}=R_{\odot}$, which results in $\bar{n}(\mathrm{LPG} \mid$ cmulti $) \simeq 3$.
} 
host LPGs with $P \gtrsim 10^{3}$ days.

\subsection{Different Mutual Inclinations or Occurrence Rates in Single- and Multi-transiting Systems?}

Table 4 also shows that the fraction of transiting LPGs in KOIs with only one inner transiting candidate is smaller than the above multiple-candidate case by an order of magnitude. While we suffer from the small statistics, the fact suggests that the term $p$ (tra|LPG, cmulti) $\bar{n}$ (LPG $\mid$ cmulti) $/ P_{\mathrm{LPG}}$ in Equation (8) is smaller for (a part of) the single-candidate sample. This means that either (A) mutual inclination of the LPG relative to the inner planet may be larger, (B) the occurrence rate of the LPG may be smaller, or (C) the typical orbital period of the LPG may be longer, in the systems with only one inner transiting planet. If (A) is actually the case, the result supports the scenario by Morton \& Winn (2014) that a population of highly inclined multi-planet systems contributes the excess of single-transiting systems in the Kepler multiplicity statistics (Lissauer et al. 2011), which is known as the "Kepler dichotomy."

We are grateful to Masahiro Ikoma and Yasushi Suto for fruitful discussions, the Kepler team for their revolutionary data, and an anonymous referee for many helpful suggestion. H.K. is supported Grant-in-Aid for Young Scientists (B) from Japan Society for Promotion of Science (JSPS), No. 25800106. K.M. is supported by JSPS Research Fellowships for Young Scientists (No. 26-7182) and by the Leading Graduate Course for Frontiers of Mathematical Sciences and Physics (FMSP). M.A. acknowledges the support by the Advanced Leading Graduate Course for Photon Science (ALPS).
Bedell, M., Meléndez, J., Bean, J. L., et al. 2015, A\&A, 581, A34 Boisse, I., Pepe, F., Perrier, C., et al. 2012, A\&A, 545, A55

Dalba, P. A., Muirhead, P. S., Fortney, J. J., et al. 2015, ArXiv e-prints, arXiv: 1510.03430

Fabrycky, D. C., Lissauer, J. J., Ragozzine, D., et al. 2014, ApJ, 790,146

Foreman-Mackey, D., Hogg, D. W., Lang, D., \& Goodman, J. 2013, PASP, 125, 306

Hagelberg, J. 2010, in In the Spirit of Lyot 2010, 22

Lissauer, J. J., Ragozzine, D., Fabrycky, D. C., et al. 2011, ApJS, 197,8

Lissauer, J. J., Marcy, G. W., Bryson, S. T., et al. 2014, ApJ, 784,44

Mandel, K., \& Agol, E. 2002, ApJ, 580, L171

Markwardt, C. B. 2009, in Astronomical Society of the Pacific Conference Series, Vol. 411, Astronomical Data Analysis Software and Systems XVIII, ed. D. A. Bohlender, D. Durand, \& P. Dowler, 251

Marmier, M., Ségransan, D., Udry, S., et al. 2013, A\&A, 551, A90

Mayor, M., \& Queloz, D. 1995, Nature, 378, 355

Montañés-Rodríguez, P., González-Merino, B., Pallé, E., López-Puertas, M., \& García-Melendo, E. 2015, ApJ, 801, L8

Morton, T. D., \& Winn, J. N. 2014, ApJ, 796, 47

Parviainen, H. 2015, MNRAS, 450, 3233

Ragozzine, D., \& Holman, M. J. 2010, ArXiv e-prints, arXiv:1006.3727

Rowan, D., Meschiari, S., Laughlin, G., et al. 2015, ArXiv e-prints, arXiv:1512.00417

Salter, G. S., Tinney, C. G., Wittenmyer, R. A., et al. 2014, in

IAU Symposium, Vol. 299, IAU Symposium, ed. M. Booth, B. C. Matthews, \& J. R. Graham, 66-67

Seager, S., \& Mallén-Ornelas, G. 2003, ApJ, 585, 1038

Sing, D. K. 2010, A\&A, 510, A21

Tremaine, S., \& Dong, S. 2012, AJ, 143, 94

Wang, J., Fischer, D. A., Barclay, T., et al. 2015, ArXiv e-prints, arXiv:1512.02559

Winn, J. N. 2010, ArXiv e-prints, arXiv:1001.2010

Winn, J. N., \& Fabrycky, D. C. 2015, ARA\&A, 53, 409

Wu, Y., \& Murray, N. 2003, ApJ, 589, 605 
Table 1

List of the 28 Single Transit Events We Identified

\begin{tabular}{|c|c|c|c|c|c|c|c|c|}
\hline$\overline{\text { KepID }}$ & $\mathrm{KOI}^{\mathrm{a}}$ & architecture $^{\mathrm{b}}$ & Kepler mag & $\bar{T}_{c}(\mathrm{KBJD})$ & depth $(\mathrm{ppm})^{\mathrm{c}}$ & $T_{\text {eff }}(\mathrm{K})$ & log $g(\mathrm{cgs})$ & $P_{\text {Kep,min }}(\text { days })^{\mathrm{d}}$ \\
\hline 8505215 & 99.01 & $1 \mathrm{CS}+1 \mathrm{~F}$ & 13.0 & 140.0473 & 1874.2 & 4965 & 4.555 & 1450.9538 \\
\hline 9970525 & 154(new) & $1 \mathrm{~F}+1 \mathrm{~S}$ & 13.2 & 139.7277 & 1500 & 6504 & 4.355 & 1451.2732 \\
\hline 11709124 & 435.02 & $5 \mathrm{C}+1 \mathrm{CS}$ & 14.5 & 657.2698 & 8709.6 & 5937 & 4.559 & 933.7415 \\
\hline 7040629 & 671(new) & $4 \mathrm{C}+1 \mathrm{~S}$ & 13.8 & 786.7641 & 1000 & 6220 & 4.242 & 804.2469 \\
\hline 8738735 & 693(new) & $2 \mathrm{C}+1 \mathrm{~S}$ & 13.9 & 697.8591 & 1000 & 6332 & 4.472 & 893.1428 \\
\hline 6191521 & $847^{\mathrm{e}}$ & $1 \mathrm{C}+2 \mathrm{~S}$ & 15.2 & 382.9428 & 5000 & 5665 & 4.563 & $\cdots$ \\
\hline 6191521 & $847^{\mathrm{e}}$ & $\cdots$ & $\cdots$ & 1489.1858 & 5000 & 5665 & 4.563 & $\cdots$ \\
\hline 2162635 & 1032.01 & $1 \mathrm{CS}+2 \mathrm{~S}$ & 13.9 & 176.0986 & 4129.9 & 5009 & 3.755 & 1414.9127 \\
\hline 2162635 & 1032(new) & $\cdots$ & $\cdots$ & 992.3180 & 1500 & 5009 & 3.755 & 860.8054 \\
\hline 2162635 & 1032(new) & $\cdots$ & $\cdots$ & 1351.3427 & 1750 & 5009 & 3.755 & 1219.8301 \\
\hline 3230491 & 1096.01 & $1 \mathrm{CS}$ & 14.7 & 315.33083 & 9592.0 & 5606 & 4.597 & 1275.6733 \\
\hline 3218908 & 1108(new) & $3 \mathrm{C}+1 \mathrm{~S}$ & 14.6 & 766.6855 & 5000 & 5513 & 4.599 & 824.3164 \\
\hline 10460629 & $1168^{\mathrm{e}}$ & $1 \mathrm{C}+2 \mathrm{~S}$ & 14.0 & 608.26209 & 22000 & 6449 & 4.232 & $\cdots$ \\
\hline 10460629 & $1168^{\mathrm{e}}$ & $\cdots$ & $\cdots$ & 1133.28363 & 22000 & 6449 & 4.232 & $\ldots$ \\
\hline 10287723 & 1174.01 & $1 \mathrm{CS}$ & 13.5 & 393.5944 & 1474.9 & 4500 & 4.572 & 1197.4066 \\
\hline 3962440 & 1208.01 & $1 \mathrm{CS}$ & 13.6 & 249.4412 & 3245.9 & 6487 & 4.397 & 1341.5601 \\
\hline 11342550 & 1421.01 & $1 \mathrm{CS}$ & 15.3 & 524.2844 & 9515.3 & 5923 & 4.445 & 1066.7171 \\
\hline 10187159 & 1870 (new) & $1 \mathrm{C}+1 \mathrm{~S}$ & 14.4 & 604.1071 & 6000 & 5185 & 4.440 & 986.8949 \\
\hline 5942949 & 2525 (new) & $1 \mathrm{C}+1 \mathrm{~S}$ & 15.7 & 1326.1614 & 20000 & 4806 & 4.564 & 1179.4970 \\
\hline 3241604 & 2824(new) & $1 \mathrm{~F}+1 \mathrm{~S}$ & 15.3 & 1263.4172 & 5000 & 5881 & 4.516 & 911.0409 \\
\hline 1717722 & $3145^{\mathrm{e}}$ & $2 \mathrm{C}+1 \mathrm{~S}$ & 15.7 & 1439.1972 & 20000 & 4812 & 4.607 & 1269.6764 \\
\hline 10284575 & 3210 (new) & $1 \mathrm{~F}+1 \mathrm{~S}$ & 11.9 & 740.72319 & 6000 & 7296 & 4.103 & 850.27786 \\
\hline 8636333 & $3349^{\mathrm{e}}$ & $1 \mathrm{C}+1 \mathrm{~S}$ & 15.3 & 271.8903 & 1500 & 6247 & 4.489 & 1319.1091 \\
\hline 6145201 & 3475(new) & $1 \mathrm{~F}+1 \mathrm{~S}$ & 13.0 & 789.1 & 1000 & 6517 & 4.382 & $\ldots$ \\
\hline 3558849 & $4307^{\mathrm{e}}$ & $1 \mathrm{C}+1 \mathrm{~S}$ & 14.2 & 279.9881 & 5000 & 6175 & 4.440 & 1311.0131 \\
\hline 4042088 & 6378(new) & $1 F+2 S$ & 13.4 & 617.65 & 17500 & 6475 & 4.234 & 973.34652 \\
\hline 4042088 & 6378(new) & $\ldots$ & $\ldots$ & 661.74 & 4300 & 6475 & 4.234 & 929.2648 \\
\hline 9581498 & 7194(new) & $1 \mathrm{~F}+1 \mathrm{~S}$ & 14.2 & 685.43 & 1000 & 5795 & 4.435 & 905.5850 \\
\hline
\end{tabular}

aLetters in the "architecture" column have the following meanings: C=planet candidates listed in the KOI catalog as "CANDIDATE"; $\mathrm{F}=$ planet candidates listed in the KOI catalog as "FALSE POSITIVE"; S=STE we identified; CS=both C and S (i.e. STEs that are already listed in the KOI catalog as planet candidates).

$\mathrm{b}$ "(new)" after the KOI name indicates the transit-like events that were not reported in the KOI catalog.

${ }^{\mathrm{c}}$ Approximate depths from the visual inspection for new candidates. For the known candidates, catalog values are listed instead.

${ }^{\mathrm{d}}$ The $P_{\mathrm{Kep}, \min }$ is the minimum possible orbital period of the STE candidates determined from the absence of other transits in the existing Kepler data. Here we neglect the possibility that other transits fell into the data gaps.

eThese events are not listed in the KOI catalog, but have been independently reported in the recent paper by Wang et al. (2015).

Table 2

Parameters of the STE Candidates Derived from Our MCMC Analysis

\begin{tabular}{|c|c|c|c|c|c|c|c|c|c|c|c|}
\hline KOI & $\rho_{*}(\mathrm{~g} / \mathrm{cc})$ & $u_{1}+u_{2}$ & $u_{1}-u_{2}$ & $\bar{T} T_{c}(\mathrm{KBJD})$ & $P$ (day) & cosi & $\bar{~} k=R_{p} / R_{*}$ & $\overline{b^{\dagger}}$ & $\overline{T T(\mathrm{hr})^{\dagger}}$ & $\bar{\tau} \tau(\mathrm{hr})^{\dagger}$ & 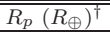 \\
\hline \multicolumn{12}{|c|}{ (Uehara Objects of Interest) } \\
\hline $847(1)$ & $0.47_{-0.13}^{+0.12}$ & $0.609_{-0.063}^{+0.064}$ & 0.166 & $382.9428_{-0.0046}^{+0.0049}$ & $930_{-380}^{+430}+\dagger$ & $0.00277_{-0.00057}^{+0.00098}$ & $0.0676_{-0.0030}^{+0.0021}$ & $0.79_{-0.12}^{+0.047}$ & $15.69_{-0.37}^{+0.34}$ & $2.9_{-1.0}^{+0.87}$ & $5.6 \pm 2.5$ \\
\hline $847(2)$ & $0.48_{-0.14}^{+0.12}$ & $0.611_{-0.060}^{+0.065}$ & 0.166 & $1489.1858 \pm 0.0046$ & $840_{-300}^{+380 \dagger \dagger}$ & $0.0029_{-0.00061}^{+0.0011}$ & $0.0680_{-0.0022}^{+0.0018}$ & $0.776_{-0.082}^{+0.048}$ & $15.66_{-0.28}^{+0.27}$ & $2.73_{-0.72}^{+0.75}$ & $5.7 \pm 2.5$ \\
\hline 1108 & $0.96_{-0.29}^{+0.22}$ & $0.68 \pm 0.11$ & 0.106 & $766.6855_{-0.0035}^{+0.0031}$ & $1160_{-430}^{+760}$ & $0.00099_{-0.00044}^{+0.00039}$ & $0.0665_{-0.0019}^{+0.0024}$ & $0.46_{-0.29}^{+0.18}$ & $19.23_{-0.26}^{+0.28}$ & $1.62_{-0.33}^{+0.61}$ & $5.5 \pm 1.9$ \\
\hline 671 & $0.96_{-0.36}^{+0.15}$ & $0.63_{-0.13}^{+0.14}$ & 0.0118 & $786.7641_{-0}^{+0}$ & $7700_{-2500}^{+2900}$ & $0.00021_{-0.00015}^{+0.00012}$ & $0.02675_{-0.00062}^{+0.00066}$ & $0.29_{-0.22}^{+0.24}$ & $39.03_{-0.37}^{+0.41}$ & $1.13_{-0.096}^{+0.34}$ & $3.9 \pm 2.5$ \\
\hline 693 & $0.34_{-0.18}^{+0.14}$ & $0.71 \pm 0.19$ & 0.00304 & $697.8591_{-0}^{+0}$ & $\begin{array}{l}980_{-470}^{+520} \\
-x_{-4}\end{array}$ & $0.0012_{-0.00082}^{+0.0014}$ & $0.0325_{-0.0010}^{+0.0012}$ & $0.32_{-0}^{+0}$ & $26.58_{-0.46}^{+0.62}$ & $0.96_{-0.098}^{+0.42}$ & $3.5 \pm 1.5$ \\
\hline 435 & $1.10_{-0.20}^{+0.18}$ & $0.596_{-0.062}^{+0.064}$ & 0.21 & $657.2698_{-0.0015}^{+0.0065}$ & $910_{-230}^{+210}$ & $0.00152_{-0.8}^{+0 .}$ & $0.0860_{-0.0017}^{+0.0013}$ & $0.579_{-0.097}^{+0.061}$ & $15.73 \pm 0.15$ & $2.05_{-0.30}^{+0.29}$ & $7.8 \pm 3.3$ \\
\hline 2525 & $3.8_{-1.1}^{+1.00}$ & $0.64_{-0.39}^{+0.282}$ & 0.467 & $1326.1614 \pm 0.0015$ & $1200_{-390}^{+540}$ & $0.00140_{-0.00031}^{+0.00052}$ & $0.16_{-0.0091}^{+0.12}$ & $0.88_{-0.046}^{+0.19}$ & $5.3_{-0.17}^{+1.0}$ & $5.12_{-0.99}^{+0.30}$ & $12.7 \pm 1.3$ \\
\hline $1421^{*}$ & $1.40 \pm 0.34$ & $0.61_{-0.11}^{+0.12}$ & 0.0907 & $524.2844 \pm 0.0025$ & $2230_{-740}^{+960}$ & $0.00068_{-0.00019}^{+0.00025}$ & $0.0913_{-0.0031}^{+0.0018}$ & $0.54_{-0.22}^{+0.092}$ & $20.14_{-0.28}^{+0.32}$ & $2.63_{-0.62}^{+0.54}$ & $10.2 \pm 4.5$ \\
\hline \multicolumn{12}{|c|}{ ("Misfit" Singles) } \\
\hline 4307 & $0.90_{-0.58}^{+0.73}$ & $0.62_{-0.10}^{+0.11}$ & 0.0329 & $279.9881_{-0 .}^{+0 .}$ & $610_{-400}^{+460}$ & $0.0009_{-0.0}^{+0.0}$ & $0.0641_{-0.0010}^{+0.0013}$ & $0.23_{-0.17}^{+0.22}$ & $16.85 \pm 0.18$ & $1.14_{-0.060}^{+0.23}$ & $6.8 \pm 2.6$ \\
\hline 3349 & $0.73_{-0.43}^{+0.26}$ & $0.66_{-0.28}^{+0.22}$ & 0.00906 & $271.8903_{-0.0088}^{+0.0086}$ & $510_{-290}^{+480}$ & $0.0015_{-0.00096}^{+0.0019}$ & $0.0363_{-0.0017}^{+0.0020}$ & $0.37_{-0.28}^{+0.32}$ & $16.48_{-0.54}^{+0.43}$ & $0.69_{-0.099}^{+0.50}$ & $3.8 \pm 1.4$ \\
\hline 1870 & $1.0_{-0.42}^{+1.4}$ & $0.78_{-0.10}^{+0.11}$ & 0.329 & $604.1071 \pm-0.0015$ & $190_{-85}^{+270}$ & $0.0014_{-0.0011}^{+0.0023}$ & $0.0722_{-0.0013}^{+0.0019}$ & $0.20_{-0.16}^{+0.21}$ & $11.05_{-0.14}^{+0.16}$ & $0.83{ }_{-0.036}^{+0.16}$ & $7.2 \pm 6.3$ \\
\hline $1208^{*}$ & $0.65_{-0.30}^{+0.31}$ & $0.52 \pm 0.16$ & -0.0243 & $249.4412_{-0.0016}^{+0.0015}$ & $65_{-36}^{+73}$ & $0.0096_{-0.0040}^{+0.0078}$ & $0.0570_{-0.0025}^{+0.0016}$ & $0.66_{-0.40}^{+0.10}$ & $7.29_{-0.12}^{+0.14}$ & $0.75_{-0.32}^{+0.38}$ & $7.0 \pm 3.8$ \\
\hline $1174^{*}$ & $2.63_{-0.42}^{+0.43}$ & $0.79_{-0.21}^{+0.15}$ & 0.564 & $393.5944_{-0.0032}^{+0.0038}$ & $310_{-88}^{+370}$ & $0.00152_{-0.00098}^{+0.00053}$ & $0.0343_{-0.0016}^{+0.0029}$ & $0.42_{-0.31}^{+0.40}$ & $8.98_{-0.28}^{+0.23}$ & $0.37_{-0.069}^{+0.33}$ & $2.72 \pm 0.15$ \\
\hline $1096^{*}$ & $2.68_{-0.35}^{+0.36}$ & $0.43_{-0.33}^{+0.40}$ & 0.183 & $315.3283_{-0.0017}^{+0.0016}$ & $700_{-160}^{+190}$ & $0.00268_{-0.00041}^{+0.00065}$ & $0.20_{-0.083}^{+0.26}$ & $1.06_{-0.12}^{+0.29}$ & $3.66_{-0.10}^{+0.14}$ & $3.66_{-0.10}^{+0.13}$ & $16.6 \pm 5.9$ \\
\hline \multicolumn{12}{|l|}{ (EB-like) } \\
\hline $1168(2)$ & $\begin{array}{l}0.500-0.040 \\
0.548+0.040\end{array}$ & $\begin{array}{l}0.910-0.059 \\
0.982^{+0.014}\end{array}$ & 0.008801 & $\begin{array}{c}\text { 608.26209-0.00043 } \\
1133.28363 \pm 0.00040\end{array}$ & $\begin{array}{l}130-9.4 \\
117_{-12}^{+11}\end{array}$ & $\begin{array}{l}0.0155-0.0017 \\
0.0168^{+0.0029}\end{array}$ & $\begin{array}{l}0.42^{-0.082} \\
0.43_{-0.14}^{+0.14}\end{array}$ & $\begin{array}{l}1.20-0.094 \\
1.21^{+0.16}\end{array}$ & $\begin{array}{l}4.700-0.046 \\
4.0 .028\end{array}$ & $\begin{array}{l}1.710-0.046 \\
4.700^{+0.028}\end{array}$ & $61 \pm 31$ \\
\hline
\end{tabular}

$\dagger$ Posteriors of these parameters were derived from those of the fitted parameters. The error in $R_{p}$ is based on the posterior of $k$ and the error in $R_{\star}$ reported on the CFOP website.

${ }^{\dagger}$ Assuming that the two STEs are due to the same object, we obtain $P=1106.243 \pm 0.007$ days from their interval.

* Prior on the mean stellar density was adopted for these systems since they do not host transiting planets other than the STE candidate. 
Table 3

List of the "Misfit" Singles and the Minimum Values of Eccentricity Required

\begin{tabular}{cccccc}
\hline \hline & & fiducial values & \multicolumn{3}{c}{ most conservative values } \\
KOI & $P_{s}($ days $)$ & $P_{\text {Kep }, \text { min }}$ (days) & $e_{\min }$ & $P_{\text {Kep }, \min }$ (days) & $e_{\min }$ \\
\hline 4307 & $610_{-400}^{+460}$ & 1311 & 0.25 & 335 & $\cdots$ \\
3349 & $510_{-290}^{+480}$ & 1319 & 0.31 & 532 & $\ldots$ \\
1870 & $190_{-85}^{+270}$ & 987 & 0.50 & 494 & 0.31 \\
$1208^{*}$ & $65_{-36}^{+73}$ & 1342 & 0.77 & 340 & 0.50 \\
$1174^{*}$ & $310_{-88}^{+370}$ & 1197 & 0.42 & 411 & $\ldots$ \\
$1096^{*}$ & $700_{-160}^{+190}$ & 1276 & 0.20 & 487 & $\ldots$ \\
\hline
\end{tabular}

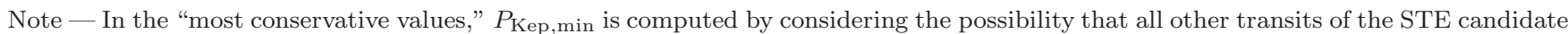
are hidden in the data gaps, although such a possibility is quite low in some cases, depending on the candidate.

* No transiting planet candidates other than the STE one are known for these systems.

Table 4

Occurrences of Transiting LPGs

\begin{tabular}{lccc}
\hline \hline & \# of transiting LPG candidates & total \# of KOIs ${ }^{\dagger}$ & fraction of transiting LPG candidates $^{-3}$ \\
\hline systems with multiple inner candidates & 4 & 695 & $6 \times 10^{-3}$ \\
systems with only one inner candidate & 2 & 2963 & $7 \times 10^{-4}$ \\
\hline
\end{tabular}

$\dagger$ Objects dispositioned as false positives are not counted as planet candidates.

\section{APPENDIX}

Here we show the PDCSAP light curves of all the 28 STEs we found (Figure 5) and the phase-folded transit light curves of the inner candidates simultaneously fitted with the 16 STE light curves in Figure 2 (Figure 6). 

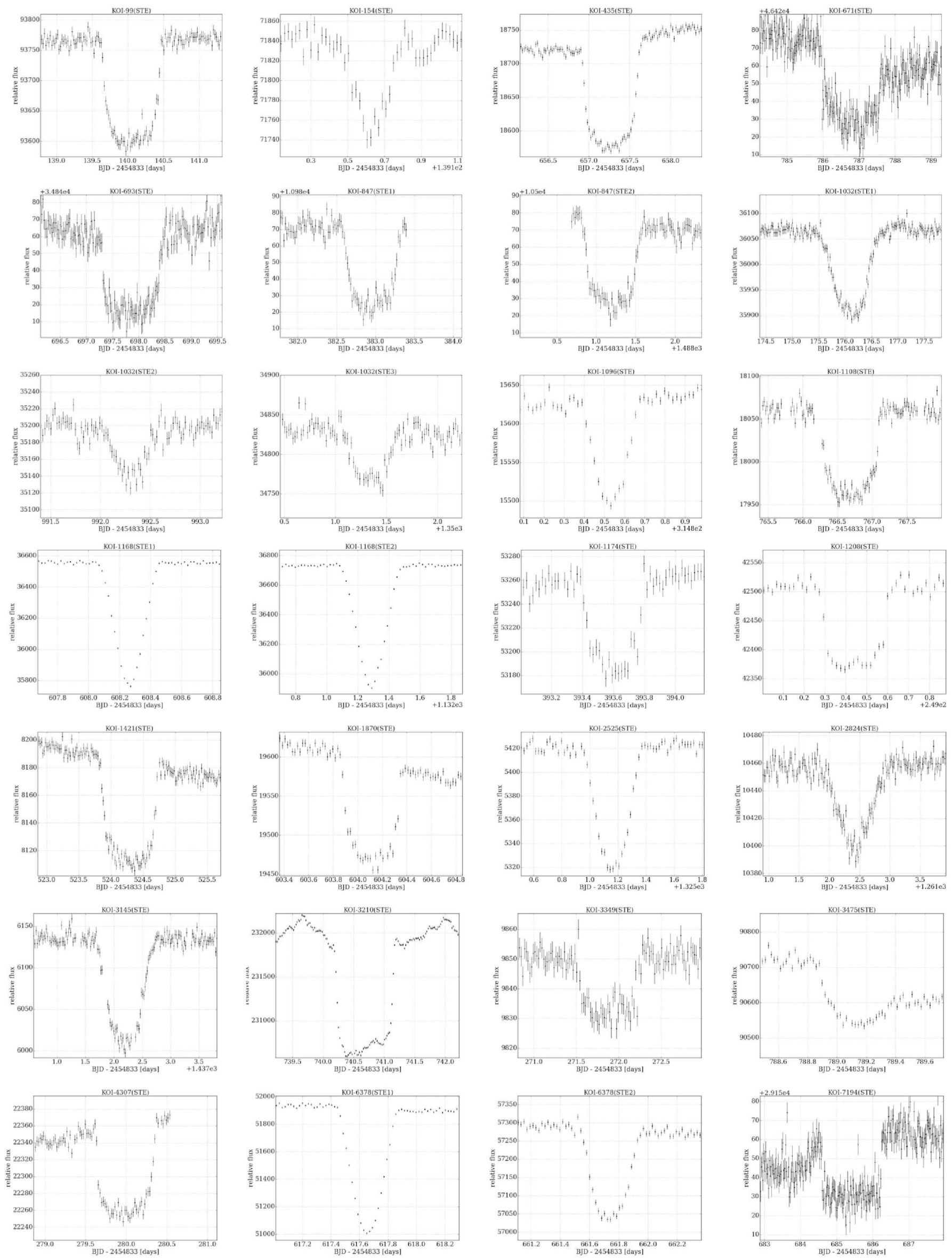

Figure 5. PDCSAP light curves of all the 28 STEs in Table 1 we identified. 

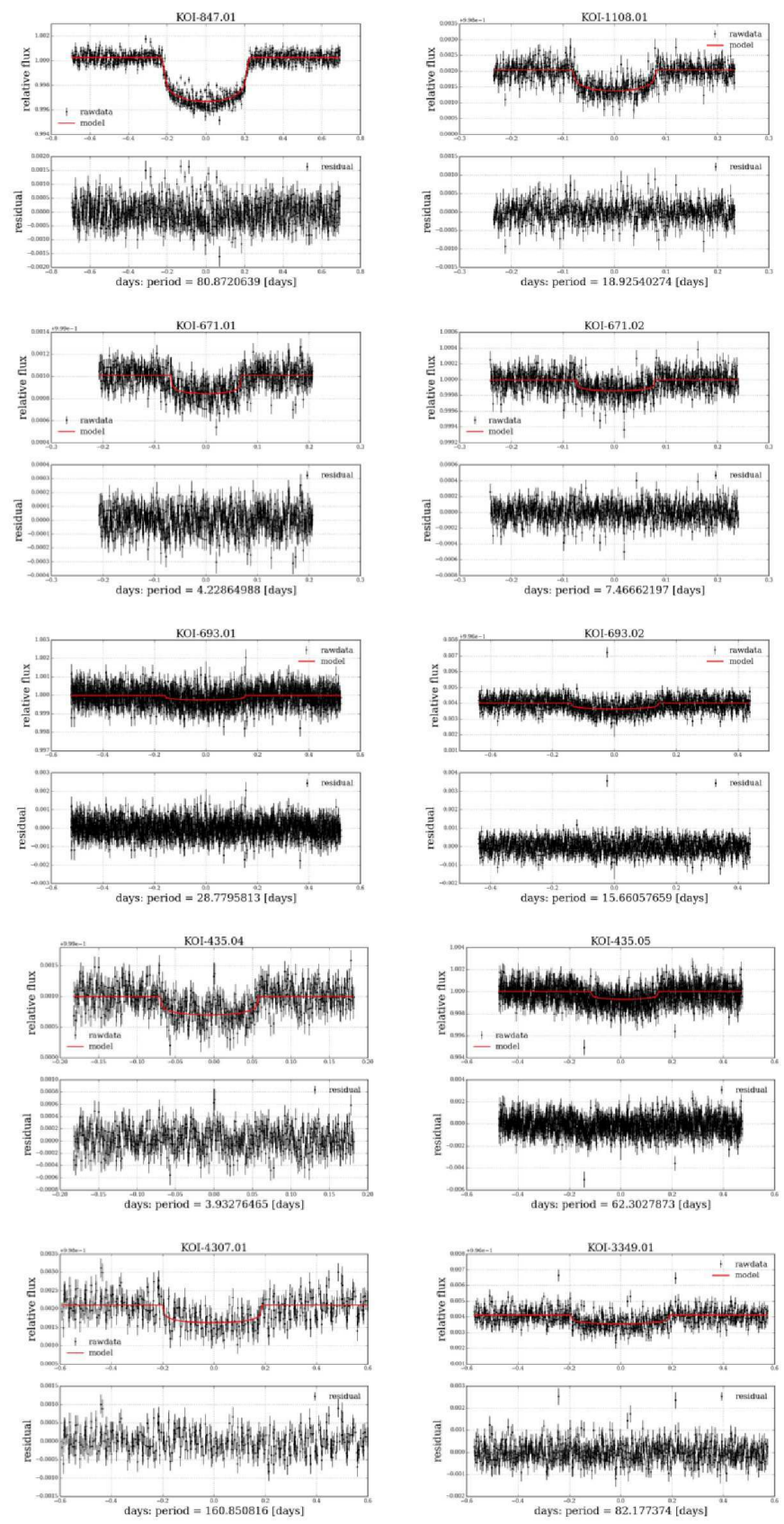
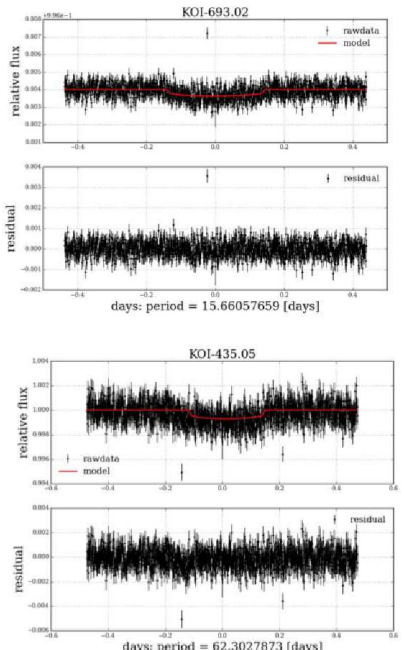

days: period $=62.3027873$ [days $]$

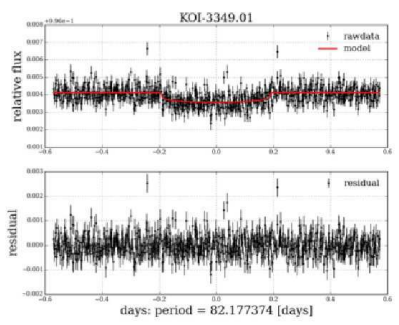

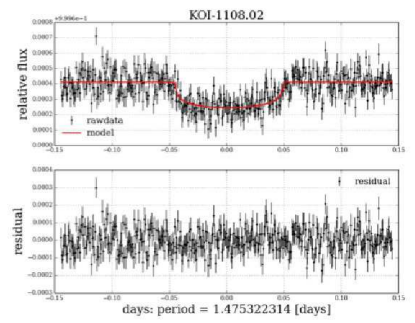


Figure 6. Fit to the phase-folded transits of the inner candidates for 10 of the systems without asterisks in Table 2 error bars show the binned fluxes and the red solid line is the best-fit model obtained from the joint fit with the STE candidates. 